\title{
Automatic Voltage Regulator based Fuzzy Logic
}

\author{
Ahmed M. H. AL-Yousif \\ Department of Electrical Engineering \\ Engineering College \\ Mosul University \\ Mosul, Iraq
}

\begin{abstract}
The objective of this paper is to design a nonlinear excitation controller of the synchronous generator based on fuzzy logic to maintain the system voltage within permissible limits by adjusting the excitation of the synchronous generator (SG) according to variation of load. The main benefit of this paper which exists in designing and implementing a fuzzy logic controller (FLC) is the ease and flexibility of the design, so FLC was applied to design an Automatic Voltage Regulator (AVR) of a synchronous generator.
\end{abstract}

The FLC constitutes a powerful tool to improve the quality of the electric voltage delivered by the SG. Simulation was carried out using Matlab Program to get the output response of the system. Simulation results, showed that the overall system response had been improved significantly when using FLC based PID controller.

\section{Keywords}

Fuzzy Logic Controller FLC, Automatic Voltage Regulator AVR, Synchronous Generator SG, Proportional-Integral Derivative PID controller.

\section{INTRODUCTION}

This paper describes the design of FLC based AVR of the SG. The proposed design regulates the armature voltage of the SG under varying load conditions by controlling the excitation current fed to the excitation field windings of the SG [1], where the task of FLC based AVR is to hold the terminal voltage magnitude of the alternator at a specified level by adjusting the SG output voltage in order to maintain it at a relatively constant value and hence maintaining the continuous supply of power with an acceptable quality, to all of the system consumers, keeping the balance between the power generation and power demand, avoiding the power system voltage instability which would seriously affect the

\section{EXCITATION SYSTEM}

When the power system consumer loads change, the SG terminal voltage would also change, thus the voltage of the SG can be kept constant by varying the SG excitation field current according to the load variation. This is known as security of the power system [2]. The power system stability and quality would thus depend on proper operation of the AVR of the SG, according to wide range operating conditions of the generators and loads [3].

FLC based AVR is considered as a modern type of AVR, where FLC has been suggested as a possible solution to use linguist information and avoid a complex system mathematical model, with accepted performance under different operating conditions [4]

This is achieved by comparing the output voltage with a reference voltage and from the difference (error); it makes the necessary adjustments in the field voltage to bring the output voltage closer to the required value [5].

It is impossible to obtain optimal operating conditions through a fixed excitation controller due to the fact that any power system is a typical large dynamic system and its dynamic behavior has great influence on the voltage stability. Thus, a model of an automated FLC based control strategy is proposed to be used in this research for controlling the generators voltage. [6]

In this paper the design and implementation of an FLC is described for controlling and regulating the output voltage of an SG. An automated FLC strategy is presented for controlling the armature voltage of SG by varying the excitation field current in real time. The FLC proposed system takes into account the voltage difference between the instantaneous output voltage and the reference rated voltage of the generator. It will also take into account the rate of change of voltage error. The controller will make an intelligent decision on the amount of field current that should be applied to the generator excitation field windings in order to keep the output voltage at its rated value. This control algorithm will be implemented on a Digital Signal Processor [7].

excitation filed current control method. The excitation systems have taken many forms over the years of their evolution. A schematic picture of an SG with its excitation filed current system, is shown in Figure 1, [2].

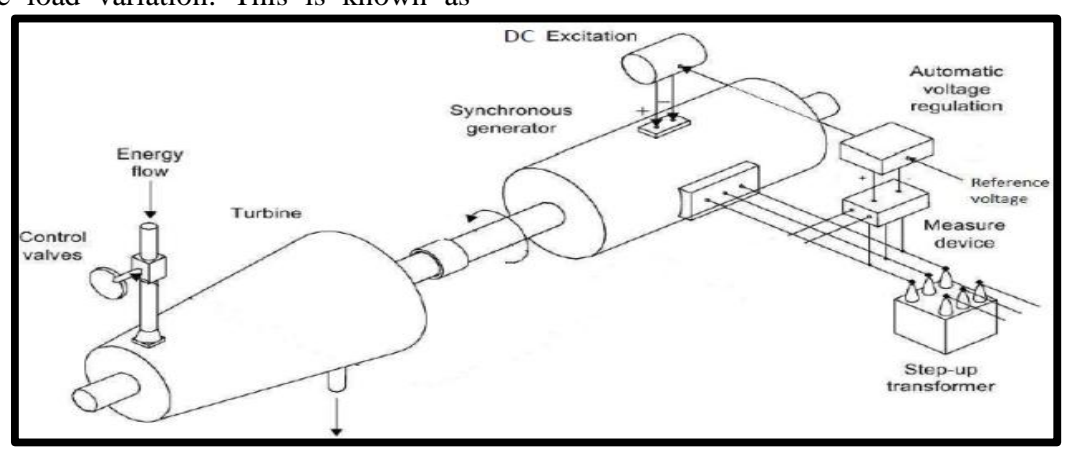

Fig 1: Schematic diagram of a synchronous machine with excitation system control. 
Enhancing power system stability and maintaining voltage regulation are two main goals which have been simultaneously addressed by nonlinear control design of the excitation field current system [8].

Excitation field current control of SG is a very important issue in the operation and control of any power system. The main control function of the excitation field current control system is to regulate the generator terminal voltage that is accomplished by adjusting the field current with respect to the variation of the terminal voltage. Classical methods that make use of linear models for designing controllers are valid only on small variation around an operating point [7]

\section{AUTOMATIC VOLTAGE REGULATOR (AVR)}

Synchronous generators SGs play a very important role in the stability of power systems. Electric power system stability requirement is increasing along with the growth of load. Thus, a robust reliable AVR systems is needed to enhance the voltage stability. One of the major auxiliary parts of SG is AVR to regulate the terminal voltage of the SG whenever any drop in terminal voltage occurs due to sudden or accidental change in loading or at any fault occurrence. system. The function of the AVR is to compare a reference voltage with a sensed and stepped down transformed and rectified terminal voltage or the error signal [4].

The function of the AVR is to maintain the generator terminal voltage at a preset value. Any change in the terminal voltage from the desired value is detected and is used as the actuating signal to control the excitation [9]. The AVR also improves the transient stability of the power

\section{FUZZY LOGIC CONTROLLER (FLC)}

FLC is a non-mathematical decision algorithm that is based on an operator's experience. This type of control strategy is suited well for non- linear systems such as the synchronous generator, which exhibits non-linearity between the field current in and the armature voltage out [8]. Basically, FLC is a multivalued logic that allows intermediate values to be defined between conventional evaluations like true/false, yes/no, high/low, etc. Notions like rather tall or very fast can be formulated mathematically and processed by computers, in order to apply a more human-like way of thinking in the programming of computers. A fuzzy system is an alternative to traditional notions of set membership and logic that has its origins in ancient Greek philosophy. The fuzzy logic implementation has received a lot of attention in the recent years because of its usefulness in reducing the model's complexity in the problem solution, by linguistic terms employment which deal with the causal relationship between input and output constraints [4].

FLC are rapidly becoming a viable alternative to classical controllers. The reason for this is that FLC can closely imitate human control processes. FLC technology enables the implementation of engineering experience and experimental results in designing embedded systems.

One of the main advantages of using a FLC strategy is that it allows a model-free estimation of the system. In other words, the designer does not need to state how the outputs depend mathematically upon the inputs [1]. FLC based on the fuzzy set theory provides a useful tool for converting the linguistic control from the expert knowledge into automatic control rules [10]. By using fuzzy automatic rules from the heuristic or mathematical strategies, complex processes can be controlled effectively in many situations. But the most important and difficult point is how to obtain the proper control rules for a given system.

The fuzzy controller is composed of the following four elements:

1- A rule-base: (A set of If-Then rules), which contains a fuzzy logic quantification of the expert's linguistic description of how to achieve good control.

2- An inference mechanism: (Also called an "inference engine" or "fuzzy inference" module), which emulates the expert's decision making in interpreting and applying knowledge about how best to control the plant.

3- Fuzzification interface: Which converts controller inputs into information that the inference mechanism can easily use to activate and apply rules[9]. Fuzzifier converts a numerical variable into a linguistic label.. In a closed loop control system, the error (e) between the reference voltage and the output. Based on the inputs, FLC takes an intelligent decision on the amount of field voltage to be applied which is taken as the output and applied directly to the field winding of generator. Triangular membership functions were used for the controller [5].

Fuzzy logic architecture: The block diagram of fuzzy controller as shown in Fig. 5.

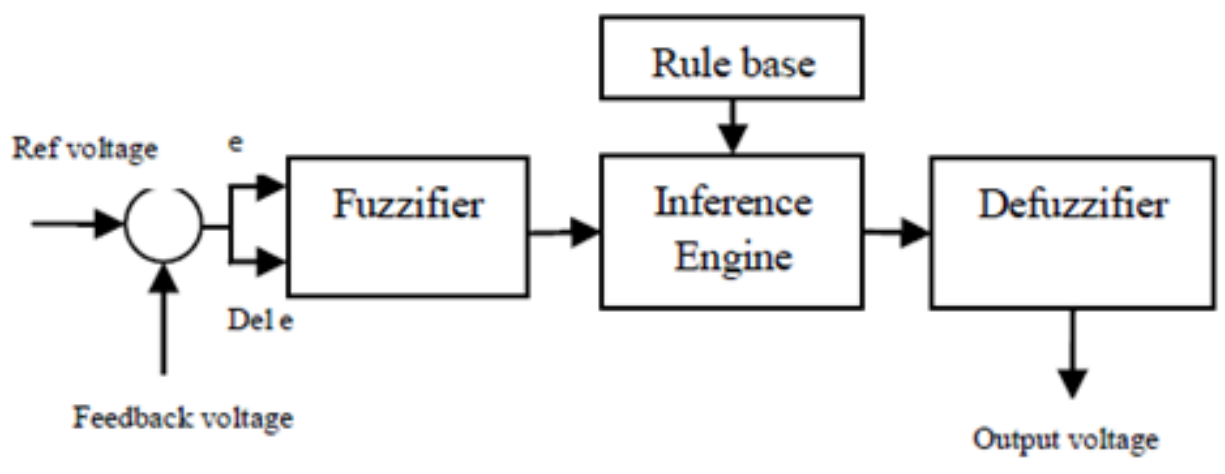

Fig 2: The general structure of Fuzzy Logic Controller. 


\section{SIMULATION AND RESULT ANALYSIS}

Simulation is carried out by the aid of MATLAB Simulink. The Simulink based SG model without an AVR is shown in figure (3) was simulated, with a load variation ranging from
$100 \%$ to $300 \%$ of its specified value, where the terminal voltage is obtained is as shown in figure (4). .

The Simulink based SG model with an FLC based AVR is shown in figure (5) was simulated, with a load variation ranging from $100 \%$ to $300 \%$ of its specified value, where the terminal voltage is obtained is as shown in figure (6).

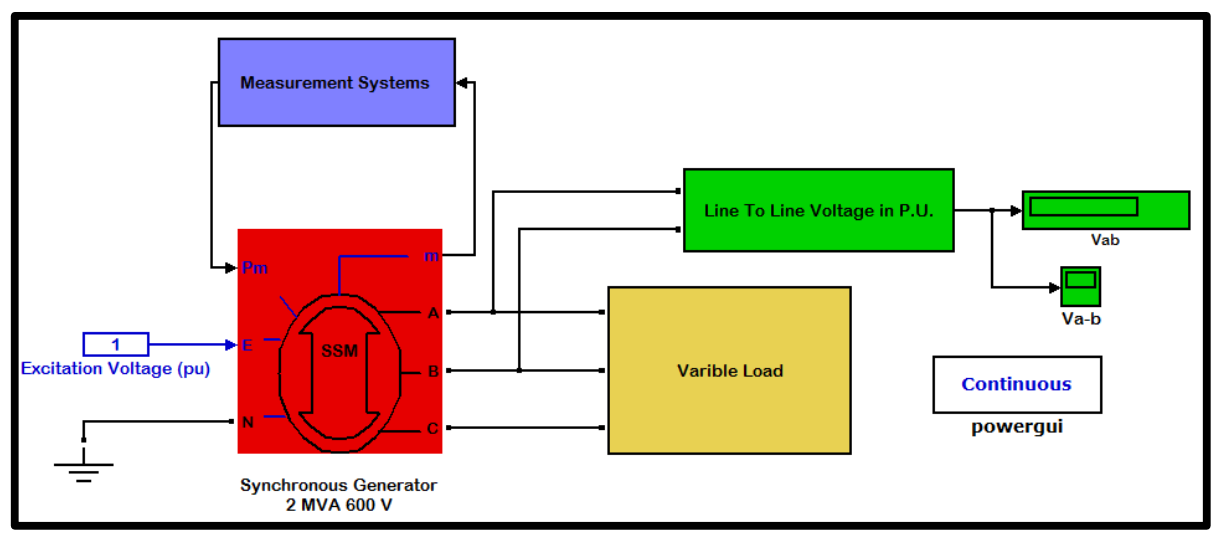

Fig 3: Effect of load variation on SG terminal voltage without FLC based AVR

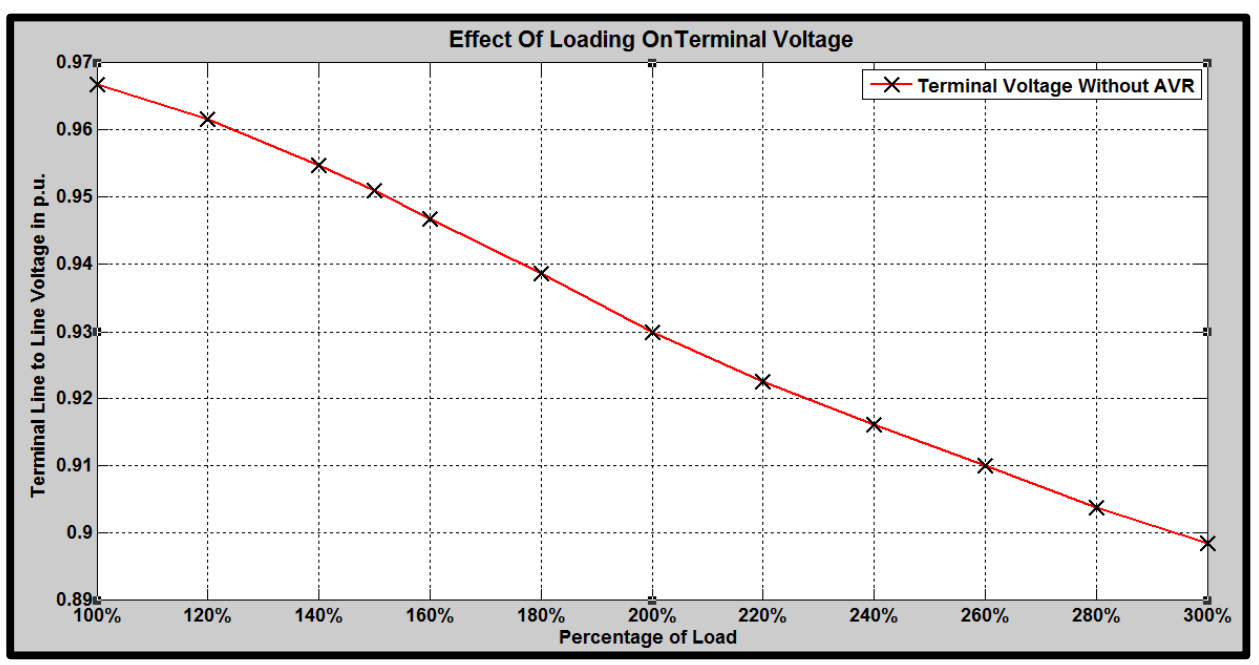

Fig 4: Effect of loading on SG terminal voltage without FLC based AVR.

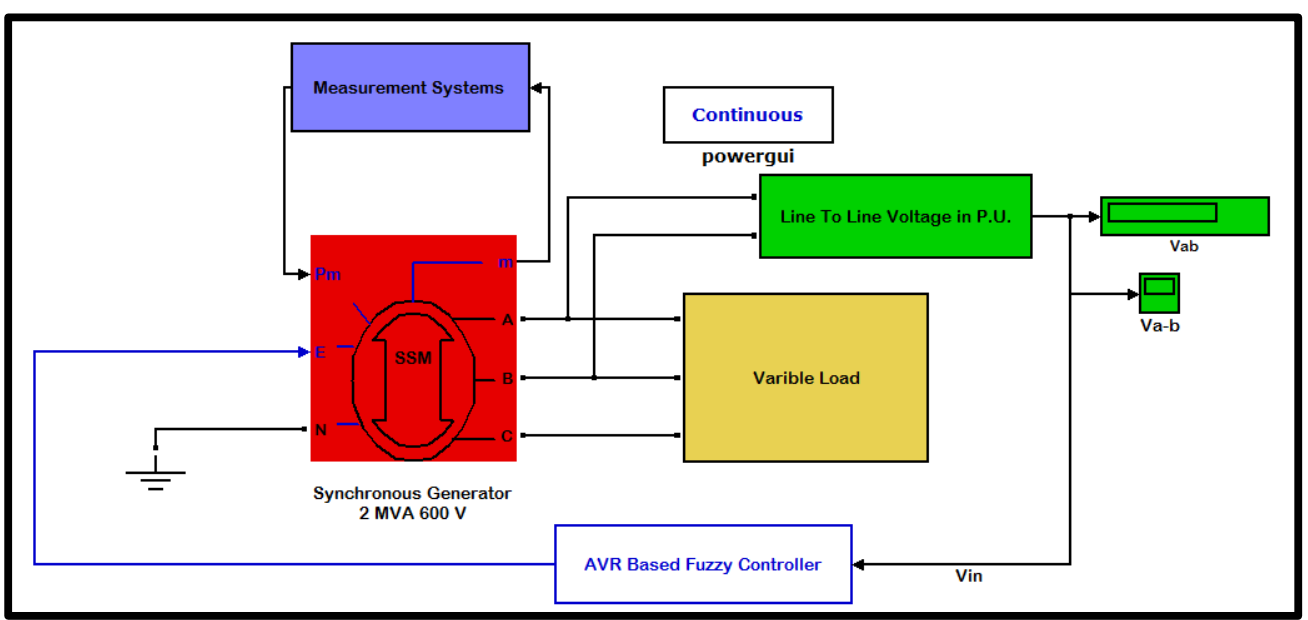

Fig 5: Effect of load variation on SG terminal voltage with FLC based AVR. 


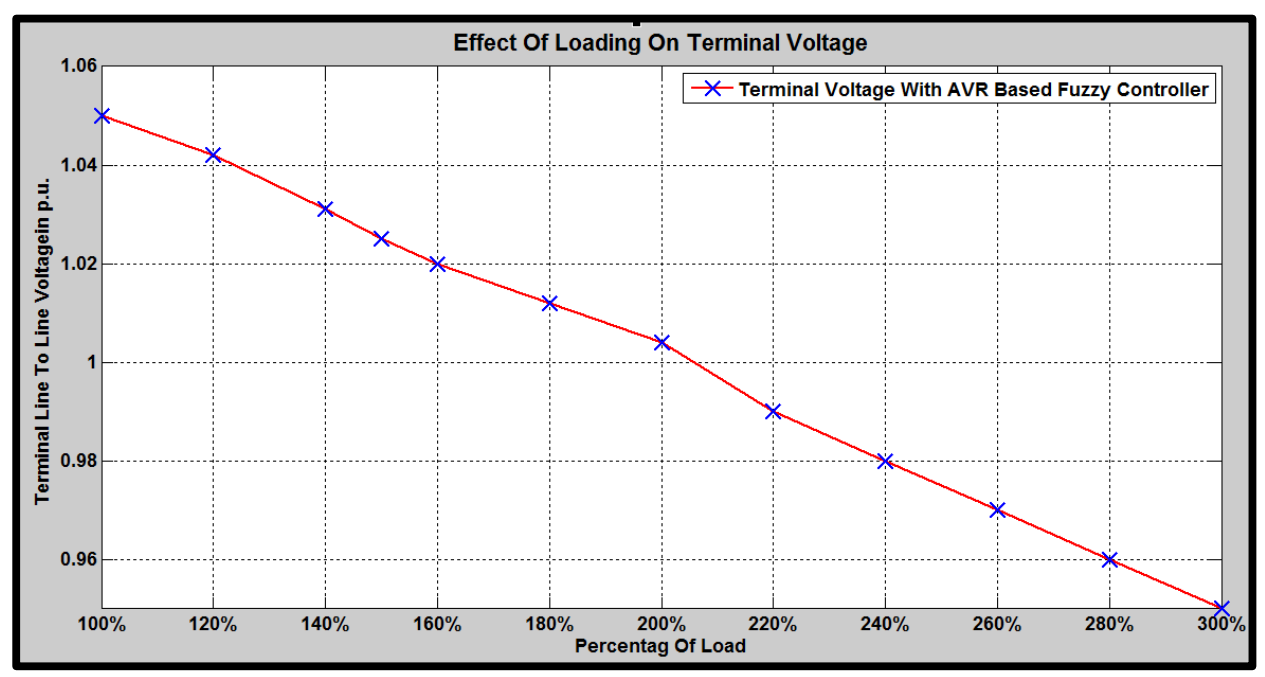

Fig 6: Effect of loading on SG terminal voltage with FLC based AVR.

\section{CONCLUSIONS}

In this paper the design and modeling of an FLC based AVR for an SG to control its voltage is discussed. The controller is to regulate the output voltage of an SG by varying the excitation field current that is to be supplied to the excitation field windings of the SG. This is accomplished by an FLC system, with the main benefit existing in the ease flexibility of the design.

\section{REFERENCES}

[1] B.J. LaMeres and M.H. Nehru." A Fuzzy Logic-Based Synchronous Generator Volt Regulator Optimized with A Genetic Algorithm". Electrical Computer Engineering Department. Montana State University. Bozeman, MT59717.

[2] Aberbour Juba ,2015. "Particle Swarm Optimization Based PID Parameters Tuning for the Automatic Voltage" MSc. Thesis. Ministry of Higher Education and Scientific Research, University M'Hamed BOUGARA Boumerdes, Institute of Electrical and Electronic Engineering, Department of Power and Control.

[3] A. Darabi, S.A. Soleamani and A. Hassannia, 2008." Fuzzy Based Digital Automatic Voltage Regulator of a Synchronous Generator with Unbalanced Loads". American J. of Engineering and Applied Sciences.

[4] Srinivas Singirikonda, G. Sathishgoud, M. Harikareddy, 2014. "Transient Stability of A.C Generator Controlled By Using Fuzzy Logic Controller" Journal of Engineering Research and Applications www.ijera.com ISSN: 2248-9622, Vol. 4, Issue 3(Version 1).

[5] Niketa N, Shantharama Rai.C 2013. "Design and Modeling of Fuzzy Logic Based Voltage Controller for an Alternator ". International Journal of Recent
In the present study, FLC based AVR was applied to improve the SG terminal voltage. From the result, it is concluded that the overall system response was improved when adding an FLC based AVR, where Figure 6, shows better performance of the AVR. It is also worthwhile to notice that FLC based AVR performance is neither too fast, nor too slow, but moderate.

Technology and Engineering (IJRTE) ISSN: 2277-3878, Volume-2, Issue-2.

[6] Clara Valdez, 2013. "Voltage Stabilizer of Generator Output Through Field Current Control Using Fuzzy Logic". MSc. Thesis. Faculty of Electrical \& Electronic Engineering, University Tun Hussien Onn Malaysia.

[7] Brock J. LaMeres. "Design and Implementation of a Fuzzy Logic-Based Voltage Controller For Voltage Regulation of a Synchronous Generator". Montana State University, Bozeman, MT 59717.

[8] Hui Liu, Junjian Qi, Peijie Li, and Jianhui Wang "Decentralized Voltage and Power Regulation Control of Excitation and Governor System with Global Asymptotic Stability" Members, IEEE.

[9] Naeim Farouk and Liu Sheng, 2012. :" Design and Implementation of a Fuzzy Logic Controller for Synchronous Generator" Maxwell Scientific Organization.

[10] Mugdesem Tanrioven, Erdin Gokalp and Celal Kocatepe, 1996. "Active and Reactive Power Adjustment of a Synchronous Generator Connected to Infinite Bus Systems by Fuzzy Logic Controller". Mathematical \& Computational Applications, Vol. 1, No.1, pp 105-112. 